\title{
THE ASTROPHYSICAL JOURNAL
}

\author{
AN INTERNATIONAL REVIEW OF SPECTROSCOPY AND \\ ASTRONOMICAL PHYSICS
}

VOLUME 91

APRIL 1940

NUMBER 3

\section{SOME PROBLEMS CONCERNING THE STRUCTURE \\ AND DYNAMICS OF THE GALACTIC SYSTEM AND \\ THE ELLIPTICAL NEBULAE NGC 3115 AND 4494}

J. H. OORT

\begin{abstract}
After a general introduction, in which some features of the density distribution and velocity distribution are discussed in connection with the possibility that the galaxy may have a spiral-like structure, the author attempts in the third section to elucidate the relation between density distribution and distribution of peculiar velocities. It is shown that if the large-scale unevenness in density found in a recent study is accepted, it is natural to expect a deviation of the stream vertex from the longitude of the galactic center of similar order as the deviation actually observed.

The distribution of light in the nearly spherical nebula NGC 4494 and in the strongly flattened elliptical nebula NGC 3 II $_{5}$ has been investigated with the aid of plates taken by Dr. Oosterhoff with the 6o-inch reflector on Mount Wilson. The results, as shown in Table $\mathrm{r}$ and Figures 3 and 4, can be fairly well represented by Hubble's formula $I=I_{\mathrm{o}} /(\mathrm{I}+r / a)^{2}$, though some small but probably real deviations are indicated. The isophotes in NGC 3 II $_{5}$, of which a few typical ones are shown in Figure 5, deviate markedly from ellipses and show remarkably sharp points at the equator. The ratio of the axes varies with the distance from the center; it has a minimum for a semimajor axis of $I^{\prime}$ (see Fig. 6). The space distribution of light in NGC 3 I I $_{5}$ has been calculated; a section with isophotal surfaces is shown in Figure 7 , while the run of space density in the equatorial plane may be found in the last column of Table $\mathrm{I}$ and in the dotted curve in Figure 4. The light density near the center is of the same order as that in the center of the globular cluster $M_{3}$.

In the last section these data are considered in relation to Humason's measures of the rotation of NGC 3 II5. It is found that beyond about ro" from the center in the equatorial plane the mass density must be about constant. The actual density depends on the unknown flattening of the attracting mass, but it should be at least of the order of I40 solar masses per cubic parsec $\left(10^{-20} \mathrm{gm} / \mathrm{cm}^{3}\right)$, or 2000 times that near the sun. In this region of constant mass density the light-density diminishes with a factor of at

I This paper was presented at the symposium on galactic and extragalactic structure, held in connection with the dedication of the McDonald Observatory on May 5-8, I939.
\end{abstract}


least Io; the distribution of mass in the system appears to bear almost no relation to that of light. The total mass of the system cannot yet be estimated; it should be at least $5.10^{10}$ solar masses but may be much larger. Expressing both mass and light in the sun as unit, one finds the ratio of mass to light in the outer parts of NGC 3 II 5 to be about 250; this contrasts sharply with the value of 1.8 found in the neighborhood of the sun and suggests an extreme relative density of very faint dwarfs or interstellar material. The analysis further indicates that the peculiar velocities of the material (stellar or other) responsible for the light of the nebula must be high, between Ioo and 200 $\mathrm{km} / \mathrm{sec}$ for the parts within $a=20^{\prime \prime}$, between $\mathrm{I} 75$ and $300 \mathrm{~km} / \mathrm{sec}$ near $a=45^{\prime \prime}$, the actual values depending again primarily upon the flattening assumed for the attracting mass. The distribution of velocities perpendicular to the equatorial plane indicates a remarkably strong deviation from a normal Gaussian law (cf. Table 5). The results of this section should be regarded as quite tentative, the main uncertainty being due to the possible existence of important absorption and diffusion of light within the nebula.

\section{THE GALACTIC SYSTEM}

STRUCTURE

About the beginning of this century the outstanding problem for the explorer of the universe was that of the size and general shape of the galactic system. The Plan of Selected Areas initiated by Kapteyn in I906 was proposed principally with a view to this problem. The Plan has been successful in that it has helped to obtain a fairly reliable picture of the general features of the density distribution within a sphere of, roughly, I 500 parsecs radius around the sun. On account of the strong absorption of light near the galactic plane it has not yet been possible to make much use of it in outlining the dimensions of the system in the plane of the Milky Way, which, however, could be derived from other data, notably those on the distribution of globular clusters, and recently also of cluster-type variables, gathered by Shapley and others.

Though it can hardly be said that our knowledge concerning the general shape of the system is anything like final, yet some of the interest is shifting gradually to another phase, namely, that of detecting an eventual spiral structure. As has been pointed out by several authors, it can hardly be doubted that in all observable characteristics (such as extreme flatness, occurrence of many highly luminous stars, existence of a well-defined center) the galactic system resembles most strongly that class of extragalactic systems which invariably show a more or less well-defined spiral structure.

The view that the galaxy might have a spiral structure has been expressed almost since the first discoveries of spiral structure in nebulae. The oldest explicit reference to this seems to be in a paper 
by Stephen Alexander. ${ }^{2}$ Both he and Proctor, ${ }^{3}$ seventeen years later, tried to find support for their spiral theory and to construct to some extent the galactic spiral from the appearance of the Milky Way as inferred mainly from the observations of the Herschels. In I900, and more completely again in I913, Easton, ${ }^{4}$ apparently quite independently and unaware of these earlier suggestions, made a very careful study of the Milky Way as shown by modern visual and photographic observations, especially with a view to delineating the course of the possible spiral arms of the galactic system; he placed the center of the spiral in the direction of Cygnus.

Later developments have made it clear that this representation can hardly resemble the real structure of the galaxy, the main cause of its failure and of the failure of all attempts to find spiral-like structure being the strong and uneven absorption near the galactic plane; for there is little doubt that many of the features of the apparent Milky Way structure are determined rather by the distribution of absorbing material than by that of the stars.

With the data at present available the difficulty (which, as indicated, is essentially that of separating the effects of absorption and of real structure) can be overcome more or less satisfactorily only in regions at some distance from the Milky Way, where the total amount of absorption may be estimated from the numbers of extragalactic nebulae (and, in some cases, also from color excesses). The procedure involves two assumptions, which it has been possible to verify only to a certain extent: (I) the assumption that the distribution of faint nebulae presents no large-scale irregularities and (2) the supposition that the absorbing material is mainly confined to regions within a few hundred parsecs from the galactic plane. The available evidence on these two points is still unsatisfactory.

2 "On the Origin of the Forms and the Present Condition of Some of the Clusters of Stars and Several of the Nebulae," A.J., 2, Ior, I852.

I am indebted to Dr. Rosseland for this reference as well as for that to Proctor's paper. Dr. Rosseland has also drawn my attention to the possibility that Lord Rosse himself may have had the idea that the galaxy had a spiral structure, but so far no direct published reference to this by Lord Rosse has been found.

3 "A New Theory of the Milky Way," M.N., 30, 50, I869.

4 "A New Theory of the Milky Way," $A$ p. J., 12, 136, I9oo, and "A Photographic Chart of the Milky Way and the Spiral Theory of the Galactic System," ibid., 37, ro5, I9I3. 
The results of a study of the Selected Areas along these lines, if accepted at their face value, show that the sun is situated in a relatively sparse region, surrounded in practically all the longitudes covered by the observations by large regions where the star density within 500 parsecs of the galactic plane is higher than that near the sun. These results are certainly very tentative, and it is probable that more extensive and more adequate subsequent studies may in several regions yield considerably different densities. But the general trend of the density distribution would seem to be fairly well established. Naturally, then, the thought presents itself that the density variations found bear some relation to the spiral structures observed in extragalactic systems; but the indications are still vague and entirely qualitative.

In a flat system like the galaxy, the main results of such density analyses as the one described can be most easily represented by comparing the counted numbers of stars in a given region to the number which would be found in a standard system in which the equidensity layers are planes parallel to the galactic plane and are so arranged that they conform to the density distribution perpendicular to the galactic plane as found in the vicinity of the sun. ${ }^{5}$ The true density distribution in space can later be easily derived from the residuals found. I believe that in the present preliminary stage of this type of investigation the procedure of starting with this simple comparison has considerable advantage, because it does not involve the use of any other data than those directly observed, and at the same time it brings out the essentials of the density phenomena studied.

It has been found by several astronomers that the arms in spiral nebulae have a color corresponding to a fairly early spectral class. ${ }^{6} \mathrm{~A}$ large-scale survey of faint B- and A-type stars in our galaxy, accompanied by a systematic and precise investigation of their colors, might accordingly furnish a good opportunity for a further search for such a structure in the galactic system. Radial velocities and studies of differential galactic rotation would furnish a necessary check on

${ }^{5}$ See Vashakidse, Bull. Abastumani Obs., No. I, p. 87, 1937, and No. 2, p. 109, 1938; Oort, Ann. d'ap., I, 71, 1938, and B.A.N., No. 308, I938.

${ }^{6}$ Seares, Proc. Nat. Acad., 2, 553, I916, and Carpenter, Pub. A.S.P., 43, 294, I931; compare also an unpublished article by Seyfert on relative colors of arms and nuclei. 
the average total absorptions. Beside color excesses and galacticrotation effects, proper motions of faint stars of known spectral type may become an important help in evaluating the absorption.

\section{MOTIONS}

It may be in place here to mention a few points concerning stellar motions, which would seem to have a bearing on the problem of the internal structure of the galactic system.

So far, the most important information derived from proper motions of faint stars has been concerned with the velocity distribution, as it has been shown that this distribution remains practically identical over a large region in the galactic plane, extending to about I 500 parsecs in the northern Milky Way. Not only do the motions of stars over this entire region follow the same ellipsoidal law, but they also appear to show the same lengths for the axes of the velocity ellipsoid and thus to indicate that the "temperature" of the stellar system does not vary perceptibly throughout the region investigated.

The extremely valuable study of radial velocities of faint $\delta$ Cephei variables recently published by Joy ${ }^{7}$ adds important evidence to that already in existence on the relative motions of different parts of the galactic system. All available data concerning these motions can be adequately interpreted as indicating that the motions of the various parts are closely perpendicular to the direction of the material center of the system, as found from globular clusters $\left(l=325^{\circ}\right.$ $\pm 2^{\circ}$ p.e.) and independently also from the distribution of stars at large distances from the galactic plane ${ }^{8}\left(l=324^{\circ} \pm 3^{\circ}\right)$. From a comprehensive study of the "rotation-effects" in the radial velocities

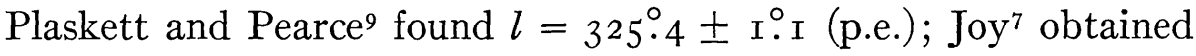
$325^{\circ} \cdot 3 \pm I^{\circ} \cdot 3$ (p.e.), while Merrill and Sanford's recent study of velocities from interstellar lines ${ }^{\text {Io }}$ yielded $329^{\circ}$. The asymmetry of high velocities and our systematic motion with respect to globular clusters and near-by extragalactic systems also point in a direction roughly $90^{\circ}$ away from the center. Considering these data together, there appears to be pretty strong evidence that the motions in the

\footnotetext{
${ }^{7}$ Ap. J., 89, 356, r939; Mt.W. Contr., No. 607.

${ }^{8}$ B.A.N., No. 308, p. 263, г938. $\quad 9$ Victoria Pub., 5, 281, г936.

г $A$ p. J., 87, I 18, I938; Mt. W. Contr., No. $5^{85}$.
} 
galactic system, at least in our surroundings, are circular around the center. If systematic motions along spiral arms exist, the arms must be very nearly circular. The data for differential rotation now extend over a disk of nearly 2500 parsecs radius around the sun, except for longitudes between $193^{\circ}$ and $27 \mathrm{I}^{\circ}$, where only a few observations are available. Apart from a (probably) local effect in the bright, relatively near-by B-type stars, the data show no evidence of a systematic expansion or contraction; Joy, for instance, finds a quite negligible value of $-3.8 \mathrm{~km} / \mathrm{sec}$ for the $\mathrm{K}$-term in his distant Cepheids.

In the bright Cygnus cloud Joy has observed two faint Cepheids, GL and V343 Cygni, which, judging from unpublished color measures recently obtained by Oosterhoff, appear to be situated at distances of about 6000 and 10000 parsecs and are yet not more than 350 and 600 parsecs from the galactic plane; MZ Cygni, at $-10^{\circ}$ latitude, likewise seems to have suffered but moderate absorption and to be about 5000 parsecs distant. If Joy's velocities for these three stars may be considered as representative for the regions in which they are situated, they show that also in this very distance part of the galactic system the motions can be satisfactorily represented by a simple rotation of this system. Computing the distances from the radial velocities, on the assumption that the residual velocities are wholly due to rotation of the galaxy, I find for GL Cygni 6700, for $V_{343}$ Cygni 9100, and for MZ Cygni 5500 parsecs, in very good agreement with the values just quoted, which were derived from the apparent magnitudes.

The available data seem as yet to be unsuited for a significant determination of the distance to the center and still less of the arrangement of mass within the galactic system. Berman, ${ }^{\text {II }}$ and, more recently, Camm ${ }^{\mathrm{I2}}$ have published evidence which they interpreted as indicating that the smallest planetary nebulae are situated at distances comparable to that of the galactic center, and some even considerably farther. They conclude that the velocities of these nebulae give, therefore, information about the dynamics of the system in regions near the center. The evidence, however, is not convincing. ${ }^{\mathrm{I} 3}$

${ }^{11}$ Lick Obs. Bull., 18, 57, 1937.

I2 M.N., 99, 71, 1938. $\quad$ г3 Cf. Oort, M.N., 99, 376, 1939. 
The large-scale irregularities indicated in the density distribution do not seem to modify the gravitational field to any observable extent, at least there is no sign of this in the differential motions. This need not cause surprise, for it can be inferred from the available data that as much as 85 per cent of the total gravitational force in our neighborhood may be considered as due to a central mass and as varying approximately as the inverse square of the distance from the center, while the rest can be adequately ascribed to a homogeneous mass extending beyond the sun.

THF DEVIATION OF THE VERTEX

Though the systematic motions of stars with small peculiar motions may be almost uninfluenced by local unevenness in the density, circumstances are quite different with regard to the peculiar velocities. It can easily be seen that a close relationship must exist between the distribution of peculiar velocities and the distribution of density in the surroundings of the sun. To simplify, let us consider stars moving exactly in the galactic plane. Size and shape of a stellar orbit may then be characterized by two quantities, for which we may take the mean distance from the center, $\bar{\omega}_{0}$, and the velocity $\Pi_{0}$ with which the star will pass the circle with radius $\bar{\omega}_{0}$ (these parameters are evidently equivalent to the parameters $I_{\mathrm{I}}$ and $I_{2}$, the energy integrals and the momentum integrals, used so extensively by Lindblad). As long as there is no instability and as long as $\Pi_{0}$ is fairly small compared to the rotational velocity of the system, the motion of a star can be described approximately as following an elliptical epicycle, the center of which moves with uniform velocity over a circle of radius $\bar{\omega}_{0}$ around the center of the system. ${ }^{14}$ Suppose, for simplicity, that the frequency distribution of $\Pi_{0}$ is the same over the whole region considered and also that the phases of the motions of the stars along the epicycles are distributed uniformly. The distribution of the centers of the epicycles will then evidently be directly related to the density distribution, while it also determines the velocity distribution at any given point.

It is well known that in a system having rotational symmetry the

${ }^{14}$ This description was first used by Lindblad in 1927 ; cf. Arkivf. mat., astr. o. fysik, 20A, No. I7, and Upsala Medd., No. 26. 
resulting distribution of velocities should be symmetrical with respect to an axis pointing to the center of the system, and this axis should be the major axis of the velocity ellipsoid.

The observations, especially those of bright stars, show, however, that the longitude of the major axis of the velocity ellipsoid deviates between $15^{\circ}$ and $20^{\circ}$ from the direction of the center. For the faint stars a deviation in the same direction is indicated, although there seems to be some evidence that it would be less pronounced for these stars.

In the representation of the system just given the most direct interpretation of the vertex deviation is that it indicates a lack of rotational symmetry in the density distribution. The assumption of an asymmetry in the density distribution of the right order to explain the deviation of the vertex is quite in line with the unevenness in the density discussed in the first section. Inversely, it might be said that the existence of the vertex deviation indicates large-scale density deviations. The relation between the two can best be illustrated by considering a simple hypothetical case.

Consider the motion of a star whose velocity is small compared to the rotational velocity of the galactic system. Let $\bar{\omega}$ be the distance from the center of the system and $\bar{\omega}_{0}$ the mean distance. If $\theta$ is the transverse velocity and $\theta_{0}$ the same velocity at $\bar{\omega}_{0}$, then the equations of motion can, in first approximation, be written as

$$
\theta \bar{\omega}=\theta_{0} \bar{\omega}_{0}, \quad \ddot{\bar{\omega}}=K_{\bar{\omega}_{0}}+\frac{\left(\bar{\omega}-\bar{\omega}_{0}\right) \partial K_{\bar{\omega}}}{\partial \bar{\omega}}+\frac{\theta^{2}}{\bar{\omega}} .
$$

Restricting ourselves again to first-order terms in $\left(\bar{\omega}-\bar{\omega}_{0}\right) / \bar{\omega}_{0}$, we can put

$$
K_{\bar{\omega} 0}=-\frac{\theta_{0}^{2}}{\bar{\omega}_{0}}
$$

and

$$
\frac{\partial K_{\bar{\omega}}}{\partial \bar{\omega}}=-2 \frac{\theta_{0}}{\bar{\omega}_{0}} \frac{\partial \theta_{0}}{\partial \bar{\omega}_{i}}+\frac{\theta_{0}^{2}}{\bar{\omega}_{0} l}
$$


so that the second equation may be reduced to

$$
\ddot{\ddot{\omega}}=-n^{2}\left(\bar{\omega}-\bar{\omega}_{0}\right)
$$

where

$$
n^{2}=2\left(\frac{\theta_{0}^{2}}{\bar{\omega}_{0}^{2}}+\frac{\Theta_{0}}{\bar{\omega}_{0}} \frac{\partial \Theta_{0}}{\partial \bar{\omega}}\right)=-{ }_{4} B(A-B)
$$

$A$ and $B$ denoting the usual constants of differential galactic rotation. We obtain, accordingly,

$$
\bar{\omega}-\bar{\omega}_{0}=a \sin n t
$$

while the difference between the star's actual angular velocity, $\omega$, and the angular velocity, $\omega_{0}$, of the center of the epicycle described by the star, is evidently given by

$$
\omega-\omega_{0}=\frac{\Theta}{\bar{\omega}}-\frac{\Theta_{0}}{\bar{\omega}_{0}}=-\frac{2 a}{\bar{\omega}_{0}} \frac{\theta_{0}}{\bar{\omega}_{0}} \sin n t
$$

In a system of co-ordinates rotating with an angular velocity $\omega_{0}$, the orbit of the star is, therefore, given by

$$
\begin{aligned}
& x=\bar{\omega}-\bar{\omega}_{0}=a \sin n t, \\
& y=\frac{2 a}{n}(A-B) \cos n t,
\end{aligned}
$$

$y$ being the transverse co-ordinate reckoned from the center of the epicycle in the direction of the rotation.

Assuming $A=+0.018 \mathrm{~km} / \mathrm{sec} \cdot$ parsec, $B=-0.013 \mathrm{~km} / \mathrm{sec} \cdot$ parsec, ${ }^{15}$ and, accordingly, $n=+0.040 \mathrm{~km} / \mathrm{sec} \cdot$ parsec, we obtain an orbit as shown in Figure r. Let us now consider a point $S$ situated in a longitude differing by $\varphi$ from that of the galactic center. We are

I5 M.N., 99, 374, I939. 
interested in the "peculiar velocity" at $S$ or, more precisely, in the difference between the original star's velocity $\Pi, \theta$ in the point $S$ and the velocity corresponding to circular motions at $S$. Denoting this

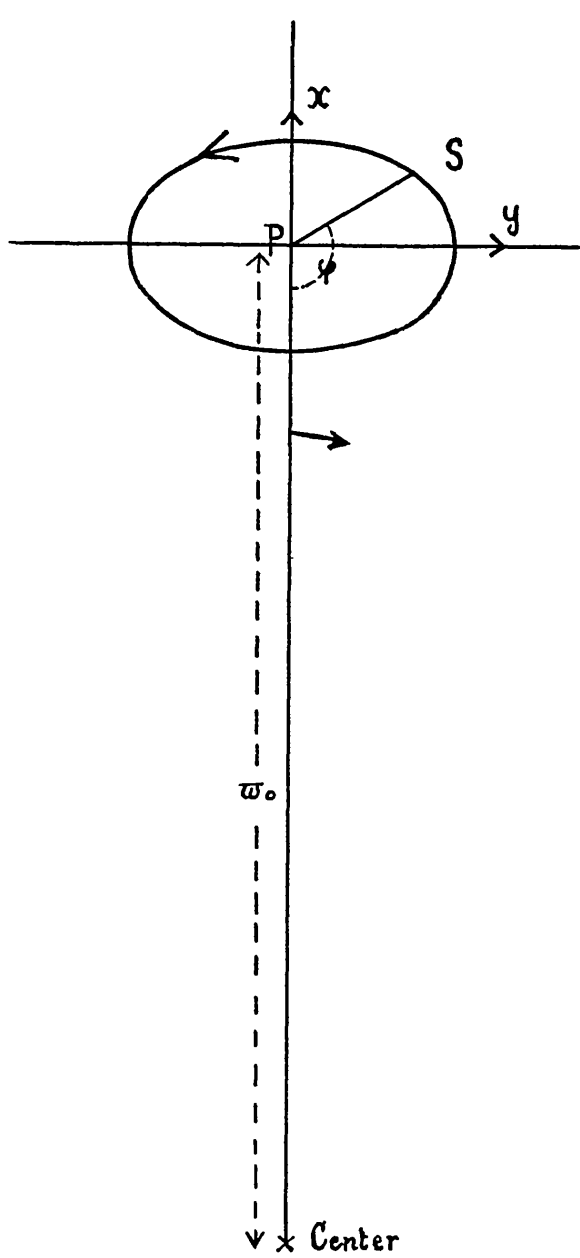

FIG. I circular velocity by $\Theta_{c, S}$, we have

$$
\Theta_{c, S}=\Theta_{0}+\frac{x \partial \Theta_{0}}{\partial \bar{\omega}}
$$

and

$$
\Theta=\theta_{0}-\frac{x \theta_{0}}{\bar{\omega}_{0}}
$$

so that

$$
\theta-\Theta_{c, S}=B x
$$

while

$$
\Pi=a n \cos n t=2 B y .
$$

If $a$ is the angle between the peculiar velocity at $S$ and the direction of the galactic center, we thus have

$$
\begin{aligned}
\operatorname{tg} a=-\frac{\left(\theta-\theta_{c, S}\right)}{\Pi} & \\
=\frac{x}{y} & =-\operatorname{ctg} \varphi .
\end{aligned}
$$

Suppose now, for an illustration, that, as seen from the sun, the density of centers of epicycles between $248^{\circ}$ and $27 \mathrm{I}^{\circ}$ galactic longitude and from 1400 to 1900 parsecs distance were twice that in the corresponding longitudes at the other side of the galactic center (between $19^{\circ}$ and $\left.42^{\circ}\right)$. At an average longitude of $260^{\circ}\left(\varphi=115^{\circ}\right)$ the distance limits considered would correspond to $x=560$ and 730 parsecs, respectively. As $\Pi=a n \cos n t=2 B x \operatorname{tg} \varphi=-0.056 x$, those stars near the sun whose epicycle centers lie in the region con- 
sidered would have peculiar velocities in the II-direction between 33 and $44 \mathrm{~km} / \mathrm{sec}$. With the aid of the foregoing equation, we find that the angles $a$ corresponding to the limits of $\varphi$ are $193^{\circ}$ and $216^{\circ}$, so

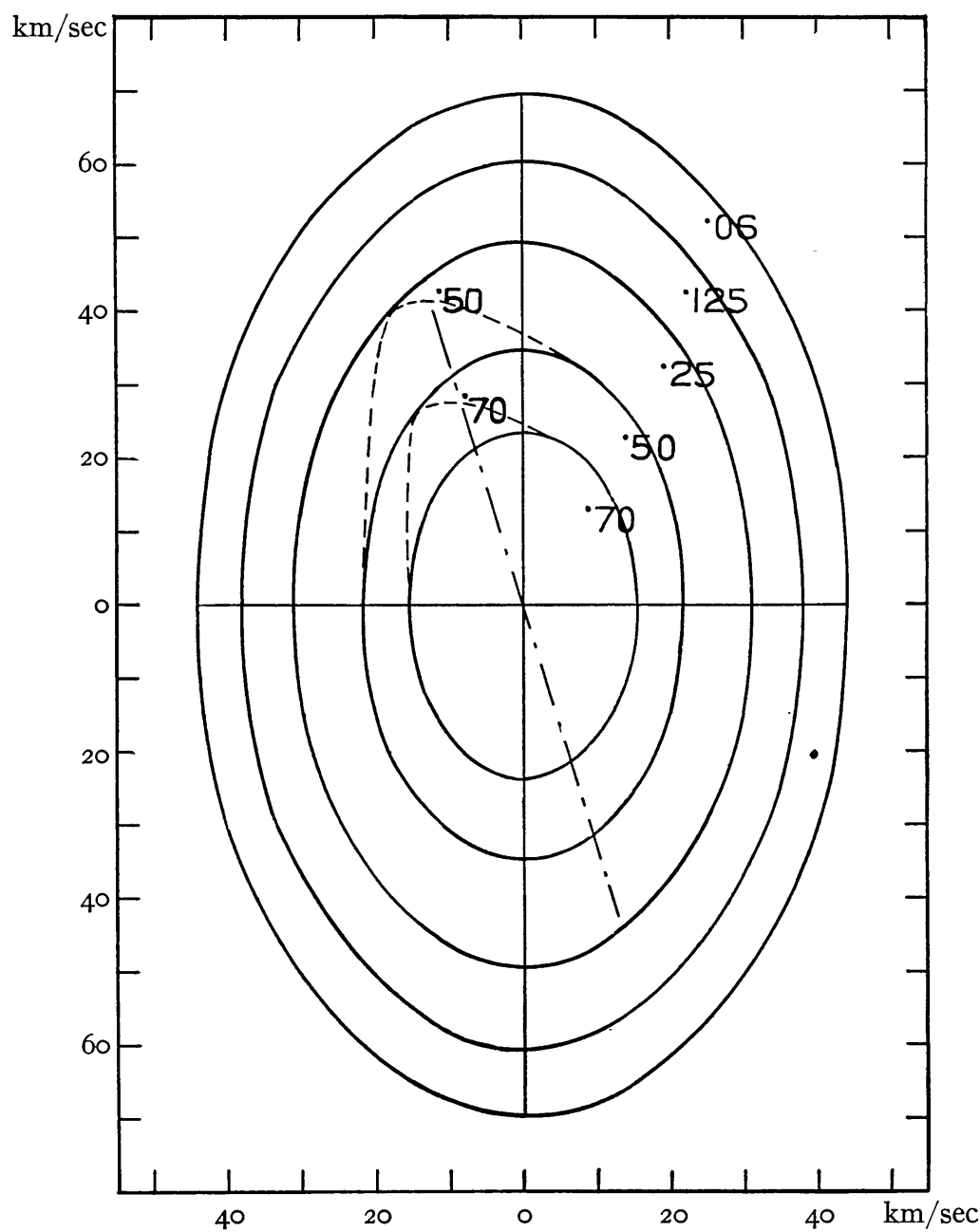

FIG. 2.-Velocity ellipsoids. The numbers attached to the ellipses indicate frequencies relative to that in the center.

that the double star density in the region considered entails a doubling of the frequency of peculiar velocities in the neighborhood of the sun between, roughly, 35 and $50 \mathrm{~km} / \mathrm{sec}$ in position angles between $193^{\circ}$ and $216^{\circ}$ as reckoned from the direction of the galactic center. The corresponding changes in the velocity distribution are roughly pictured in Figure 2. Ordinates are peculiar velocities in the 
$\Pi$-direction, abscissas those in the $\theta$-direction. The full-drawn ellipses, with their major axes pointing to the galactic center, represent lines of equal frequency in a symmetrical stellar system; the accompanying numbers indicate the relative frequencies expressed in terms of that at the center of the figure as unity. The local increase of star density considered causes the lines of equal frequency to bulge out, as shown roughly by the dotted curves, the bulges having been smoothed so as to avoid sudden transitions. The bulge extends over the range of curves with frequencies between about 0.40 and 0.16. A similar bulge for the inner curves may be obtained by an increase of the star density between 700 and 1400 parsecs in the same longitude interval by 50 per cent. Though we cannot make a very certain estimate of the vertex deviation which would be caused by the bulges considered, it is probable that it would come out at least of the same order as that observed; at the same time, it need not entail a very noticeable effect on the systematic motion of the stars considered.

The foregoing example does not pretend to resemble the actual conditions in the galactic system. It is evident that a relative excess of density in a sector opposite to the one considered would produce the same deviation in the vertex. It is also possible. and even probable, that conditions are complicated by the fact that any unevenness in the density distribution is likely to be accompanied by an unevenness in the distribution of the phases of the motions.

Available density data are still insufficient to indicate whether density deviations in the required sense exist; all that can be said, I believe, is that the data do not contradict the interpretation suggested. The only purpose of the present discussion was to illustrate the relation between velocity distribution and density distribution and to indicate the order of the deviations in the density required to explain the deviation observed in the velocity distribution. It may be well to point out, in this connection, that rather different interpretations are possible, such as, for instance, that proposed by Lindblad. ${ }^{16} \mathrm{He}$ suggests that the orbits of the stars responsible for the deviation of the vertex extend to a region where circular motions are unstable. The orbits of these stars would then be of a different character from those considered, starting in a spiral-like manner in the

${ }^{16}$ Stockholms Obs. Ann., 12, No. 4, 1936; Östen Bergstrand Festskrift, 1938. 
unstable region; it is easily conceivable how, on this model, a slight difference could occur between the velocities of outgoing and of incoming stars, which would give rise to a deviation of the vertex.

Lindblad has pointed out that the more or less sudden appearance of the strong asymmetry in the velocity distribution which is found to occur at peculiar velocities of about $65 \mathrm{~km} / \mathrm{sec}$ can also find explanation in his model. The most direct interpretation of the sudden appearance of the asymmetry would, however, seem to be that it is caused by the way in which the density in the galactic system varies in its outer regions. If considered in the same way that we considered the vertex deviation, it would mean that, after remaining roughly constant up to about 1600 parsecs in the direction of the anticenter, the density would rapidly fall off beyond this distance. Such a variation could certainly be reconciled with the available data.

\section{EXTRAGALACTIC SYSTEMS}

Accurate knowledge of the density distribution in extragalactic systems is evidently one of the prerequisites for a study of the dynamics of these systems. Apart from a few color measurements, as reported above, little or no quantitative work has yet been done on spiral nebulae proper. For the elliptical nebulae quite interesting results have been obtained by Hubble, and more recently studies on the same subject have been published by Redman and Shirley. A number of these nebulae have been studied in some detail in Leiden, with the aid of plates taken by Dr. Oosterhoff at the Mount Wilson Observatory and by the author at the Perkins Observatory. The measurements and reductions were carried out in collaboration with Mr. Kleibrink. In the following pages a report will be given on the results for two of the nebulae, NGC 4494 and NGC 3II5. The results given are provisional in several ways, and some further measurements and reductions are contemplated.

The most interesting outcome of Hubble's original investigation of the elliptical nebulae ${ }^{\mathrm{I} 7}$ was that, except for differences in flattening, all these nebulae appeared to be built on the same model. In each of the ${ }_{5}$ objects studied the surface intensity could be well represented by the formula $I=I_{\mathrm{o}} /(\mathrm{I}+r / a)^{2}$; and this is not all: it ap-

${ }^{17}$ A p. J., 7I, 23I, I930; Mt.W. Contr., No. 398. 
peared also that the "central" intensity, or rather, the parameter $I_{0}$, was nearly identical for the various nebulae; the same may hold approximately for the linear diameters. No satisfactory reason has yet been found why these nebulae should all be built on this particular model, but it is evidently a result of the greatest interest. ${ }^{18}$

PHOTOMETRY OF NGC 4494

Figure 3 shows detailed results of the intensity measures on NGC 4494 as studied at Leiden. Four plates taken by Dr. Oosterhoff with the Mount Wilson 6o-inch reflector were used, with altogether six exposures ranging from I to 60 minutes. The nebula had been classified as Eo by Hubble, but the measures indicate a ratio of 0.83 for the shortest and the longest axes. For the plot in Figure 3 various position angles were simply averaged (for most exposures tracings were made through $\mathrm{I} 2$ different position angles). The ordinates in Figure 3 show logarithms of surface intensity $I$, this being expressed in stars of magnitude 25.0 per square second of arc as unit. ${ }^{19}$ The intensity scale was determined by means of sensitometer marks; on two of the plates it was also determined from measurements of surface brightness in the rings of far interfocal images of a Selected Area exposure superimposed on the field of the nebula. These Selected Area exposures were also used for obtaining the zero points of surface brightness. The two independent determinations of the intensity scale agree very well. A partial third test is furnished by the intercomparison of the various exposures. Zero-point corrections have been applied to the different exposures in order to fit the curves together. It will be seen from the diagram that, with these corrections applied, the various exposures agree quite satisfactorily. Significant differences are found only for points derived from the faint

${ }^{18}$ It should be mentioned that Redman (M.N., 96, 588, r936; see also ibid., 98, 613, 1938) has recently found somewhat deviating results for some of the nebulae measured by Hubble. On the other hand, Miss Patterson, working at Harvard, has in an unpublished study obtained data which generally confirmed Hubble's earlier measures. The results for NGC 4494 and 3 I I 5 can be represented quite well by Hubble's formula.

19 The surface brightness expressed in magnitudes per square second of $\operatorname{arc}(H)$ is related to $I$ by the formula $B=2.5$ ( $10-\log I$ ). The relation between the surface intensity $I^{\prime}$, expressed as the number of stars of ro.o photographic magnitude per square degree (as has also been used in recent work) and that used in the present note, $I$, is $I^{\prime}=13.0 I$. 
and ill-defined part of the toe of the reduction curve. Observations corresponding to these uncertain parts have been indicated by reduced size of the characters in the figure. Abscissas in the graph are logarithms of the distance to the center in seconds of arc. The surface brightnesses plotted have not yet been corrected for the effect of

$\log I$

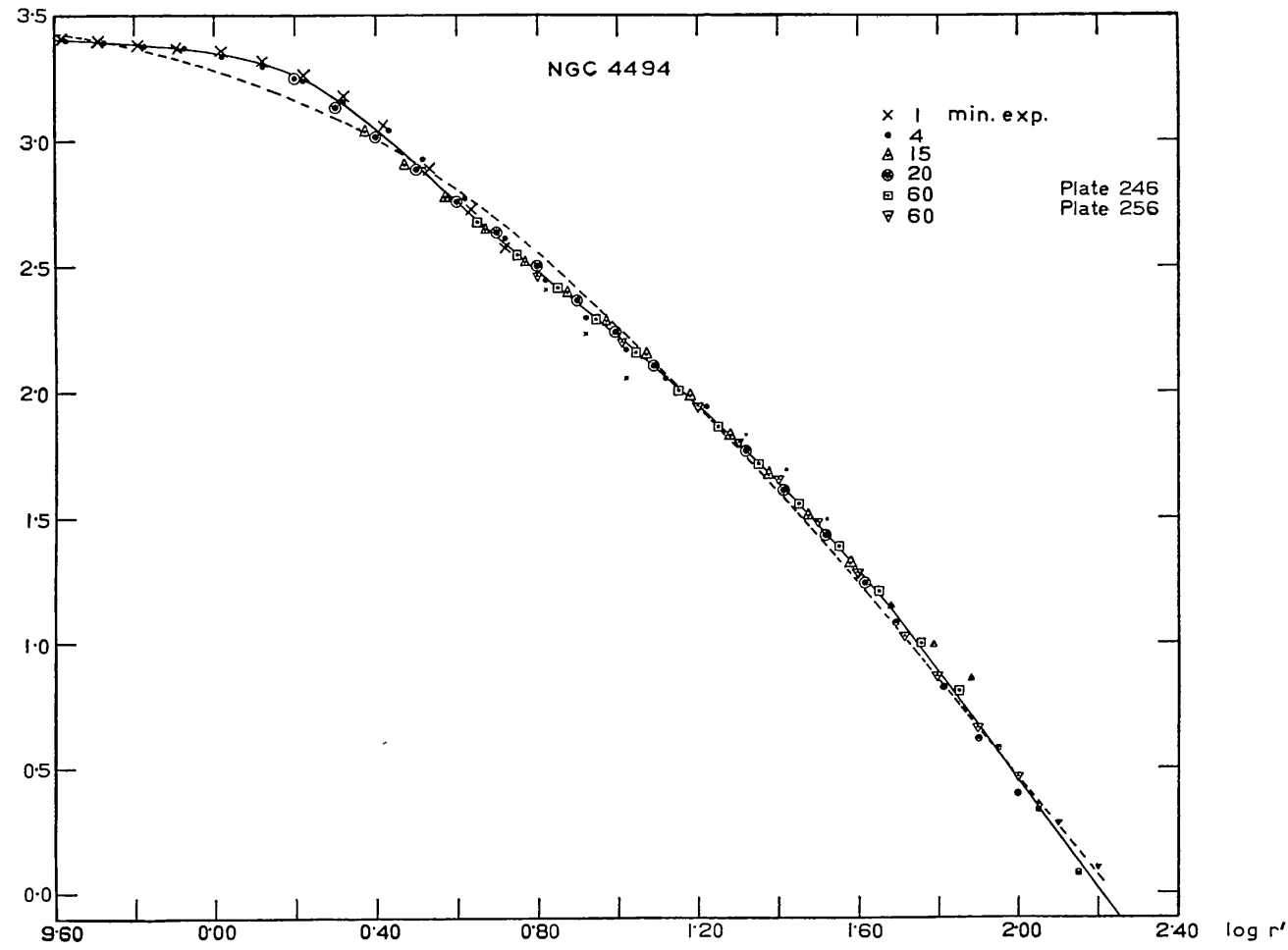

Fig. 3.-Distribution of light in NGC 4494. Ordinates are logarithms of surface intensity, expressed in stars of $25 \cdot \mathrm{m} . \circ$ per square second of arc. Abscissas are logarithms of the distance from the center in seconds. The dotted curve represents Hubble's formula $\log I=\log I_{0}-2 \log (\mathrm{r}+r / a)$, with $\log I_{0}=3.4 \mathrm{I}$ and $a=3$ ".० ( $\mathrm{I}^{\prime \prime}$ is roughly 12 parsecs).

atmospheric and instrumental diffusion of the light; this must have had a pronounced effect upon the curve to the left of $\log r=0.5$, but it is believed to have been of relatively little importance for the rest of the curve. ${ }^{20}$

${ }^{20}$ Redman and Shirley have recently shown (M.N., 98, 6I4, I938) that on their plates a measurable fraction of the light is dispersed to distances of several minutes from the source of light. Qualitatively, this has been confirmed on the Mount Wilson plates 
As will be seen from the dotted curve in Figure 3 as well as from the numbers in Table $I$, the main features of the density distribution in this nebula (which has not been measured by Hubble) can be quite well represented by Hubble's formula, with $a=3$ ". $\circ$ and

TABLE 1

Distribution OF SURFACE BRIGHTNESS IN NGC 4494 AND 3II5

\begin{tabular}{|c|c|c|c|c|c|c|c|c|}
\hline \multirow{3}{*}{$r$} & \multirow{3}{*}{ LOG $r$} & \multicolumn{2}{|c|}{ NGC 4494} & \multicolumn{5}{|c|}{$N_{G C} 3 I I 5$} \\
\hline & & \multirow{2}{*}{$\log I_{\mathrm{obs}} *$} & \multirow{2}{*}{$\mathrm{O}-\mathrm{C} \dagger$} & \multicolumn{2}{|c|}{ Major Axis } & \multicolumn{2}{|c|}{ Minor Axis } & \multirow{2}{*}{$\begin{array}{c}\text { Major } \\
\text { Axis } \\
\log \varphi \ddagger\end{array}$} \\
\hline & & & & $\log I_{\mathrm{obs}}{ }^{*}$ & $\mathrm{O}-\mathrm{C} \dagger$ & $\log I_{\mathrm{obs}} *$ & $\mathrm{O}-\mathrm{C} t$ & \\
\hline o."o......... & $-\infty$ & $3 \cdot 28$ & -0.13 & $3.6 \mathrm{I}$ & $+0: 26$ & $3.6 \mathrm{I}$ & +0.06 & +3.26 \\
\hline I.o........ & 0.0 & $3 \cdot 20$ & +.04 & $3 \cdot 4^{8}$ & $+.2 \mathrm{I}$ & $3 \cdot 46$ & +.17 & $+2.9 I$ \\
\hline $2 \cdot 5 \ldots \ldots \ldots$ & 0.4 & 2.92 & +.04 & 3.18 & +.02 & 3.07 & +.07 & +2.19 \\
\hline $4.0 \ldots \ldots$ & 0.6 & 2.65 & -.03 & 3.03 & -.03 & 2.83 & +.04 & $+\mathrm{I} .83$ \\
\hline $6.3 \ldots \ldots$ & 0.8 & $2 \cdot 3^{8}$ & -.05 & 2.89 & -.04 & 2.56 & +.03 & $+\mathrm{I} .5 \mathrm{I}$ \\
\hline ro.o........ & I.O & $2 . \mathrm{II}$ & -.03 & 2.73 & -.03 & 2.24 & .00 & +1. I9 \\
\hline $\mathrm{I}_{5} .8 \ldots \ldots \ldots$ & I. 2 & I. 83 & $+.0 I$ & $2 \cdot 55$ & $+.0 I$ & I .85 & -.07 & +0.87 \\
\hline $25.1 \ldots \ldots$ & I. 4 & I. $5 \mathrm{I}$ & +.04 & $2 \cdot 3 I$ & +.04 & I. 47 & -.09 & +0.48 \\
\hline $39.8 \ldots \ldots \ldots$ & I. 6 & I. I6 & +.06 & 2.04 & +.07 & I. I6 & -.04 & +o.0I \\
\hline$\sigma_{3} . \ldots \ldots$ & I . 8 & 0.78 & +.05 & I. $7 \mathrm{I}$ & +.07 & 0.85 & +.03 & -0.50 \\
\hline IOO.0........ & 2.0 & 0.34 & 0.00 & I. 29 & $+.0 I$ & $0.5 \mathrm{I}$ & +0.08 & - I.06 \\
\hline $\mathrm{I}_{58} 8.0 \ldots \ldots$ & 2.2 & $\ldots \ldots$ & $\ldots \ldots \ldots$ & 0.86 & -.06 & $\ldots \ldots$ & $\ldots \ldots$ & $-\mathrm{I} .65$ \\
\hline 25 I.०........ & 2.4 & $\ldots \ldots$ & $\ldots \ldots$ & 0.44 & -0.09 & $\ldots \ldots$ & $\ldots \ldots$ & -2.27 \\
\hline
\end{tabular}

* The symbols $I$ and $\varphi$ are expressed in stars of photographic magnitude 25.0 per square, or cubic, second of arc as unit.

† The columns $\mathrm{O}-\mathrm{C}$ contain the differences between the observed distributions and those computed from the formula $\log I=\log I_{0}-2 \log (\mathrm{I}+r / a)$. The constants used are NGC $4494, \log I_{0}=3.4 \mathrm{I}$; $a=3$ ! ०; NGC 3 II 5 major axis, $\log I_{0}=3.35, a=$ IO $_{2}$; NGC 3 II 5 minor axis, $\log I_{0}=3.55, a=2.84$.

$\ddagger$ The last column shows the distribution of light in space along the equatorial plane.

$\log I_{0}=3.4 \mathrm{I}$. There are, however, some minor deviations which are probably real; between 4 " and $8^{\prime \prime}$ the computed values are about 0.06 , or $\circ^{\mathrm{m}} \mathrm{I} 5$, brighter than the observed intensities, while between $25^{\prime \prime}$

used in this article by tracings through the stars BD+26 $6^{\circ} 2359\left(88^{\mathrm{m}} \cdot 4\right.$, Ao) situated only $6^{\prime}$ from NGC 4494 , and $\mathrm{BD}-6^{\circ} 3066$ ( I $^{\mathrm{m}} \cdot 2 \mathrm{pg}, \mathrm{K}_{2}$ ), I9' from NGC 3 I I $_{5}$. The magnitude of the effect may be illustrated by stating that 3 per cent of the light is dispersed to distances between $\frac{1}{2}^{\prime}$ and $2^{\prime}$ from the star. The percentage is found to be practically the same on all four long-exposure plates measured; it appears to be independent of seeing conditions and might well be due to dispersion in the plate. The amount is about four times less than that found by Redman and Shirley at the same angular distances. The effect upon the outer contours is small, even for the very condensed nebula NGC 4494 . In order to correct for it, the value of $\log I$ for $r=100^{\prime \prime}$ should be diminished by 0.04 , that for $r=63^{\prime \prime}$ by about 0.02 . 
and $50^{\prime \prime}$ they are about the same amount fainter. It should be mentioned that, on account of the uncertainty as to the true distribution of light near the center, the parameters $a$ and $I_{0}$ cannot be separated with much accuracy. A value of $a=4$ ". $\circ$, for instance (with $\log I_{0}=3.20$ ), would fit the well-observed part of the intensity distribution quite as well as the values adopted.

The distance of NGC 4494 may be estimated from its radial velocity, which was kindly communicated to me by Humason, in advance of publication. As corrections to the center of the galactic system are negligible in this case, the distance can at once be obtained by the aid of the velocity-distance relation. Using Hubble's value of $530 \mathrm{~km} / \mathrm{sec}$ per million parsecs ${ }^{2 \mathrm{r}}$ for the coefficient of expansion and Humason's velocity of $+1350 \mathrm{~km} / \mathrm{sec}$; we find $2.5 \times 10^{6}$ parsecs. The nebula is only $14^{\circ}$ away from the nucleus of the Virgo cluster; if it is a member of the nuclear part of this cluster, its distance should be $2.2 \times 10^{6}$ parsecs. $^{22}$ Assuming, provisionally, a distance of $2.4 \times \mathrm{IO}_{9}$ parsecs, $I^{\prime \prime}$ at the distance of NGC 4494 is equivalent to I 2 parsecs, and we find $a=36$ parsecs.

PHOTOMETRY OF NGC 3 I I 5

Of NGC 3 I $_{5}$, three 60-inch Mount Wilson plates taken likewise by Dr. Oosterhoff were measured, containing altogether five exposures of $60,5 \circ \frac{1}{2}$, I 5,4 , and I minutes, respectively. Details of measurements and reductions will be more fully published elsewhere in combination with the results for the other nebulae studied; we shall discuss only the main results of this study.

Several circumstances appear to make this elliptical nebula especially interesting for thorough investigation. The object is the brightest of the nebulae classified as $\mathrm{E}_{7}$ by Hubble. Its flattening, corresponding to a ratio of about 3 to I between the two axes, is the strongest observed among the nebulae classified as elliptical; this indicates that it is an extreme case, which, in the current thoughts on spiral formation, may mark the point of transition to an unstable system; it also indicates that the nebula must be seen practically edge on, and this knowledge enables us to derive the space distribution from the distribution of light as seen projected on the sky. Fur-

\footnotetext{
${ }^{21}$ The Realm of the Nebulae, p. r7o. $\quad{ }^{22}$ Ibid., p. I63.
} 
thermore, the rotation has been measured by Humason, the measurements extending to about $45^{\prime \prime}$ from the center.

Figure 4 and Table I show the results of the measures of the distribution of light in the nebula as projected on the sky, along the major and minor axes, respectively. The various exposures have

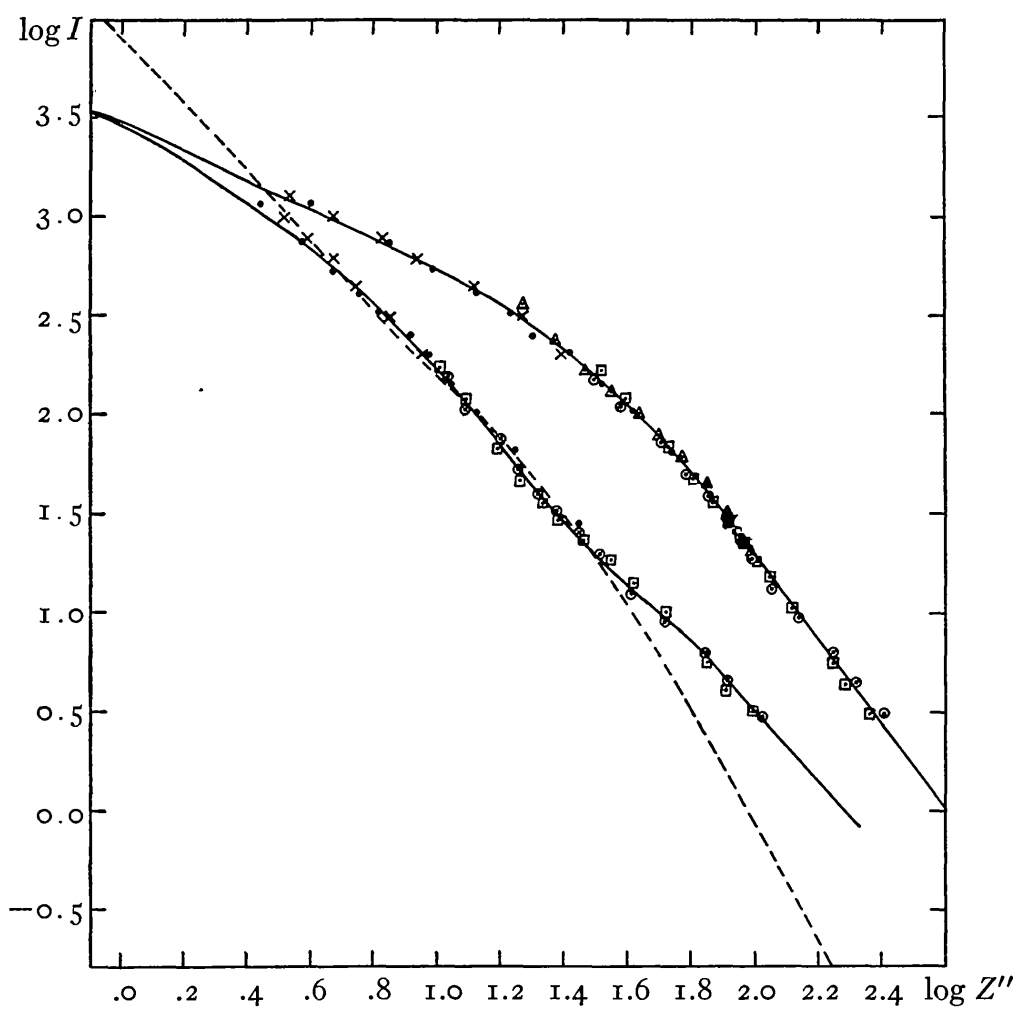

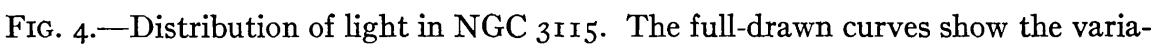
tion of surface intensity $I$ along the major and minor axes. The $\mathrm{I}$-minute exposure is represented by crosses, the 4 -minute by dots, the 15 -minute by triangles, the $50 \frac{1}{2}$-minute by circles, and the 6o-minute by squares. The dashed curve shows the variation of the space density of light, $\varphi$, along the equatorial plane. Abscissas are logarithms of the distance from the center in seconds, ordinates are logarithms of the light-intensity expressed in stars of $25^{\mathrm{m}} \cdot \mathrm{O}$ per square second for $I$ (numbers on the left) and per cubic second for $\varphi$ (numbers on the right).

again been fitted together by applying zero-point corrections. In this case a provisional correction has been applied in the region near the nucleus for the dispersion of light caused by atmosphere, instrument, and plate. This dispersion, as determined from registrograms 
through a focal star image $80^{\prime \prime}$ from the center of the nebula on the one-minute exposure, is $\pm 0.04 \mathrm{~mm}$ on the plate, or $\pm \mathrm{x}$ ".o. The corrections applied to log $I$ range from about to.I 8 in the center to -0.08 at $3^{\prime \prime}$ distance, becoming negligible beyond about $4^{\prime \prime}$. No claim is made for the numerical accuracy of these corrections; the most that can be said is that they indicate the order of the uncertainty arising from the light dispersion. It would seem that the shape of the isophotal contours (as shown in Fig. 5) and the results for the concentration of the light near the major axis (except in the central

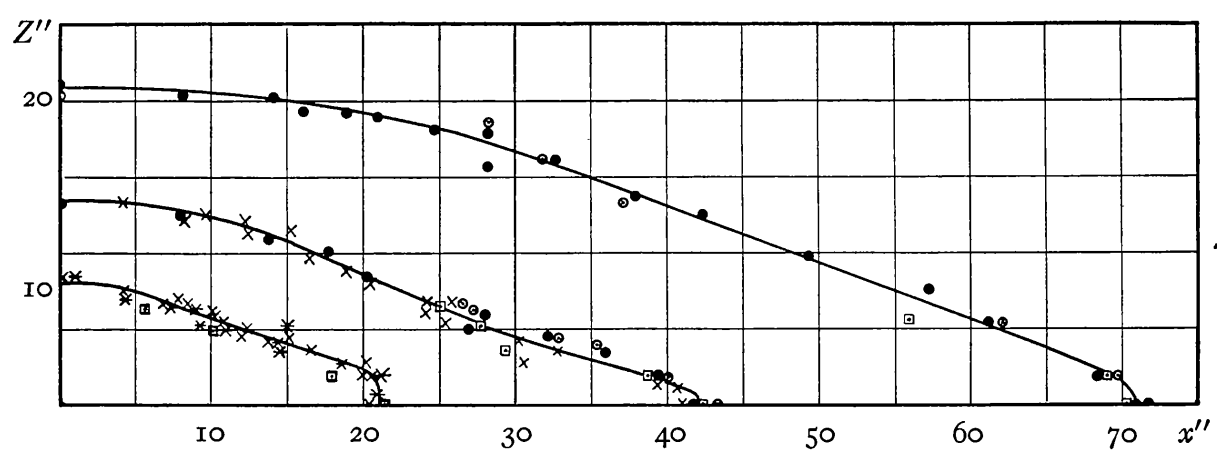

FIG. 5. - Isophotes in NGC 3 II 5 . The isophotes correspond to $\log I$ 2.4, 2.0, and r.6, $I$ being expressed in stars of $25^{\mathrm{m}}$. p per square second. The co-ordinates are given in seconds ( $I^{\prime \prime}$ is roughly 7 parsecs). The $\mathrm{I}$-minute exposure is represented by asterisks, the 4 -minute by crosses, the 15 -minute by squares, the $50^{\frac{1}{2}}$-minute by dots, and the 6o-minute by circles.

nucleus) can have been but little disturbed by the small observational dispersion.

The distributions in Table I can again be fairly well represented by Hubble's simple formula, though there are some deviations which appear to be real. The differences from the values computed by this formula, using Hubble's results for the parameters $a$ (viz., Io". 2 for the major axis and 2".84 for the minor axis), are as shown in Table I; the values of $\log I_{0}$ used for fitting the formula to the present observations are 3.35 for the major axis and 3.55 for the minor.

Isophotes for $\log I=2.4,2.0$, and 1.6 are shown in Figure 5 . They were obtained from a considerable number of tracings in directions parallel with and perpendicular to the principal axis. Special care was taken to obtain tracings near the equatorial plane and accurately parallel to it, in order to get as reliable data as possible on 
the run of the isophotes near the major axis; for this purpose each exposure was traced along the equatorial plane as well as at 0.07 and $0.20 \mathrm{~mm}$ (or I".9 and 5".4) distance on either side of it. The exact situation of the major axis had been ascertained by micrometer measures and had been indicated on the plates by dots; in this way the error of centering in the microphotometer has been reduced to something of the order of O". I, while the probable error of the orientation will not have exceeded ${ }^{\circ}$. I. The various exposures have been indicated by separate signs; the corresponding isophotes were reduced to exactly the same size by applying factors derived by equating the average of the minor and major axes of the isophotes considered to the standard values of the axes for the surface brightness concerned as read from Figure 4.

The most remarkable features of the isophotes are the remarkable sharpness with which they run into the major axis and the long, straight portions which lead down to these sharp edges-features that have also previously been remarked upon by Hubble ${ }^{23}$ his data, however, show a little less detail in the parts near the axis. There is an indication that for the inner isophote, with semimajor axis $a=$ $2 \mathrm{I}^{\prime \prime}$, and especially for isophotes still nearer to the center, the sharpness of the edge disappears, while at the same time the ratio $c / a$ of minor to major axis increases. The ratio reaches a minimum of 0.29 near $a=60^{\prime \prime}$; it increases again for the outer isophotes. The variation of $c / a$ with $\log a$ between $a=4^{\prime \prime}$ and $a=300^{\prime \prime}$ is shown in Figure 6, in which the ratios for various exposures have been plotted separately. Both the innermost isophotes (say for $a$ below $\mathrm{IO}^{\prime \prime}$ ) and the outermost isophotes (beyond $a=$ Iо०") are somewhat uncertain on account of the instrumental and atmospheric dispersion of light. The effect upon the outer isophotes has been determined on the long-exposure plates 460 and 467 by measures on the near-by tenth-magnitude star $\mathrm{BD}-6^{\circ} 3066$ (cf. also n. 20, p. 287 ); the curve gives the corrected values, while the crosses have been plotted without correction. In order to strengthen the part of the curve for large values of $a$, a number of registrograms were made of a fine roominute exposure taken by Ritchey on December 25, I9II, which was kindly put at my disposal during a stay at the Mount Wilson

${ }^{23}$ Ap. J., 71, 264, r930; Mt.W. Contr., No. 398. 
Observatory in the summer of 1939 . The results, again uncorrected for dispersion, have been plotted as asterisks in Figure 6. Hubble's earlier determinations of the axial ratio ${ }^{24}$ for the brighter parts of the nebula have also been indicated in the figure (as squares); they are slightly higher than the values derived here. No attempt has yet been made to correct the inner isophotes for the effect of light-dispersion.

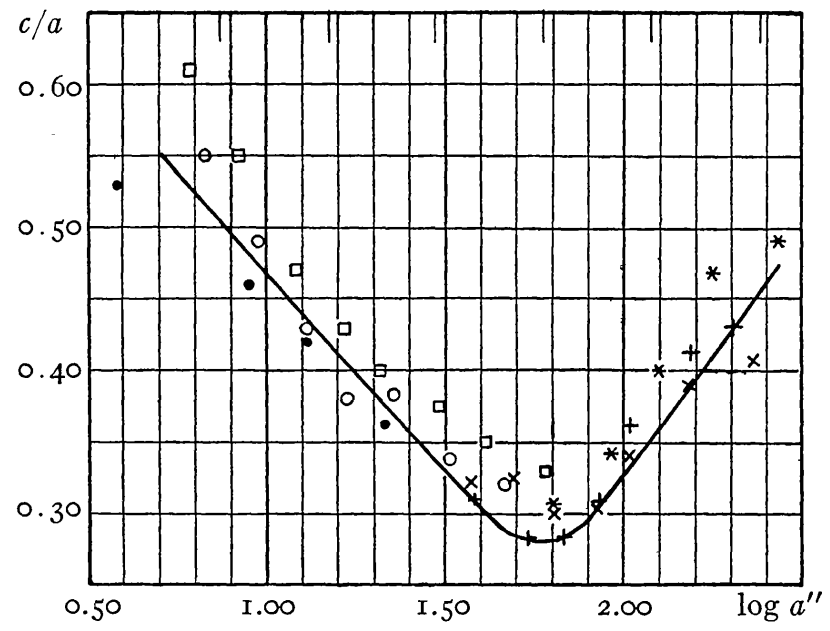

FIG. 6.-Axial ratio of isophotes in NGC 3 I I 5. Dots represent results from I-minute exposure, circles from 4 -minute, and crosses from $50 \frac{1}{2}$ - and 60-minute exposures. The asterisks have been derived from a 10o-minute exposure by Ritchey. The squares have been copied from results of short Ioo-inch exposures previously published by Hubble. The curve has been approximately corrected for the effect of light-dispersion over great distances (see text).

SPACE DISTRIBUTION OF THE LIGHT IN NGC 3 I I 5

In order to obtain at least some rough insight into the true struc-

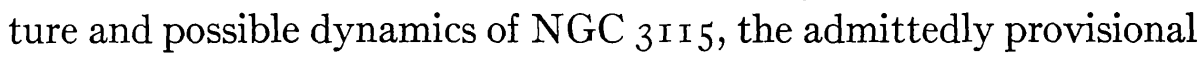
isophotes discussed in the foregoing section were used to determine the space density $\varphi$. Let $z$ denote the distance from the equatorial plane of the system; let $\varphi_{z}(\bar{\omega})$ define the space density of light at distances $\bar{\omega}$ and $z$ from the minor and major axes, respectively, expressed as the number of stars of 25.0 photographic magnitude per "cubic second of arc" at the distance of the nebula. Let $I_{z}(x)$ be the surface brightness at a distance $x$ from the minor, and $z$ from the

${ }^{24}$ Ap.J., 7r, 264, I930; Mt.W. Contr., No. 398. 
major axis. If it is assumed that the system considered has rotational symmetry around an axis perpendicular to the line of sight and that there is no absorption of light within the system, it can be shown that in any plane of constant $z$

$$
\varphi_{z}(\bar{\omega})=-\frac{\mathbf{I}}{\pi} \int_{\bar{\omega}}^{\infty} \frac{\frac{d I_{z}(x)}{d x}}{\sqrt{x^{2}-\bar{\omega}^{2}}} d x .^{25}
$$

In most of the actual integration, which was carried out by $\mathrm{Mr}$. Pels, it proved easiest to determine first, graphically, the derivative of $\log I$ with respect to $\log x$ and then to compute $d I / d x$ from these quantities. The extrapolation over the unknown outer regions is of little importance. If, in rough accordance with the observations, it is assumed that for large values of $x$ (say, beyond $X$ ) $I$ becomes of the form $c x^{-2}$ and if this formula is assumed to hold to infinity, the part of the integral expression for $\varphi$ beyond $X$ is easily seen to be

$$
\frac{c}{\pi \bar{\omega}^{3}}\left(-\frac{\bar{\omega}}{\bar{X}} \sqrt{\mathrm{I}-\frac{\bar{\omega}^{2}}{X^{2}}}+\arcsin \frac{\bar{\omega}}{X}\right) .
$$

If $X$ is taken to correspond to the outer isophotes measured, the extrapolated part is usually only a small fraction of the total. The calculations were carried out for 8 values of $z$ and were roughly checked by the inverse calculation of $I$ from $\varphi$.

Figure 7 shows a section through the isophotal surfaces as provisionally found from these calculations. The curves resemble, in general, the isophotes in the projected image, but the ratio between minor and major axis comes out somewhat larger; between $a=30^{\prime \prime}$ and $a=\mathrm{Ioo}^{\prime \prime}, c / a$ is found to be approximately 0.40 , as against 0.30 in the projected image. However, it should be remembered that the true flattening may well be somewhat stronger, as the line of sight may be slightly inclined to the true equatorial plane. The values of $\log \varphi$ corresponding to the various surfaces are indicated in the figure. In the center we find, approximately, $\log \varphi=3.26$. Taking

${ }_{25}$ The formula was first given by Von Zeipel (Ann. de l'obs. de Paris, Mém., 25, p. F30, r9o8); see also Smart, Stellar Dynamics, pp. 297-302; it is easily seen to apply to any surface of revolution the axis of which is perpendicular to the line of sight. 
the photographic light of the sun per cubic parsec as unit of density of light ${ }^{26}$ and estimating the distance of the nebula as $\mathrm{I} \frac{1}{2}$ million $\operatorname{parsecs}^{27}$ (therefore, $\mathrm{I}^{\prime \prime}=7.3$ parsecs and $m-M=25.9$ ), we find that a star of $25^{\mathrm{m}} \cdot \mathrm{O}$ per cubic second corresponds to 0.74 units; if the true distance is $\kappa$ times larger, the corresponding light per cubic parsec will be $\kappa$ times smaller. The central intensity corresponds to

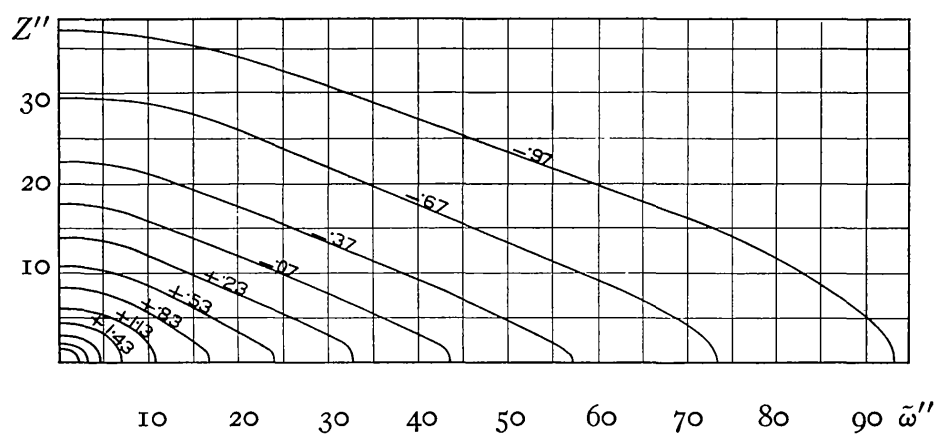

Fig. 7.-Section of isophotal surfaces in NGC $3 \times 15$. The numbers in the figure are logarithms of the light-density $\varphi$ corresponding to the respective curves, $\varphi$ being expressed in stars of $25^{\mathrm{m}} \cdot \circ$ per cubic second. For the three inner curves $\log \varphi$ is, respectively, 2.33, 2.03, and I.73; in the center $\log \varphi$ is estimated to be 3.27. Co-ordinates are in seconds ( $\mathrm{I}^{\prime \prime}$ is roughly 7 parsecs).

I 400 units. This is 30,000 times the total density of light in the part of the galactic system in which the sun is situated and is of the same order as that in the center of Messier $3 .^{28}$

${ }^{26}$ The photographic absolute magnitude of the sun was assumed to be +5.26 , in accordance with a recent discussion by Kuiper ( $A p . J ., 88,429$, r938).

${ }^{27}$ This distance is still very uncertain. The only criteria from which it can be estimated are the radial velocity, the apparent magnitude, and the size. The radial velocity, corrected to the center of the galaxy, is $+450 \mathrm{~km} / \mathrm{sec}$, corresponding to a distance of 0.8 million parsecs; however, the smallness of the velocity makes this result doubtful. From the apparent magnitude, 9.8 according to Shapley and Miss Ames, combined with Hubble's value of -15.2 for the average absolute magnitude of this class of nebulae (The Realm of the Nebulae, p. I76), a distance of 1.0 million parsecs is derived. The apparent dimensions, on the other hand, indicate a much larger distance: the average of $a_{\max }$ and $a_{\min }$ for NGC 3 I I 5 is $6 " .5$, while the average linear value of $a$ for the 7 elliptical nebulae measured by Hubble in the core of the Virgo cluster amounts to 66 parsecs; if we suppose that the average $a$ for $\mathrm{NGC}_{3 I_{5}}$ is the same, we find 2.I million parsecs for the distance. The foregoing value is thus a compromise; it should be kept in mind that the true distance may well differ from it by a factor as large as 2 in either direction.

${ }^{28}$ Hertzsprung, A.N., No. 4952, I9I8. 
The run of $\log \varphi$ with $\bar{\omega}$ in the equatorial plane of the nebula has been plotted as a dotted curve in Figure 4. The curve for $\log \varphi$ runs considerably steeper than that for $\log I$; from $\bar{\omega}=80^{\prime \prime}$ on, $\varphi$ can be expressed as $\bar{\omega}^{-3 . r}$. The smallest light density recorded is approximately $\varphi=0.01$, or 0.007 units as previously defined, or one-sixth of the light-density in the region surrounding the sun.

DYNAMICS OF NGC 3 II 5

It was hoped, originally, that the distribution of the light in extragalactic nebulae would bear resemblance to the distribution of mass and that investigations of the light-distribution might give some

TABLE 2

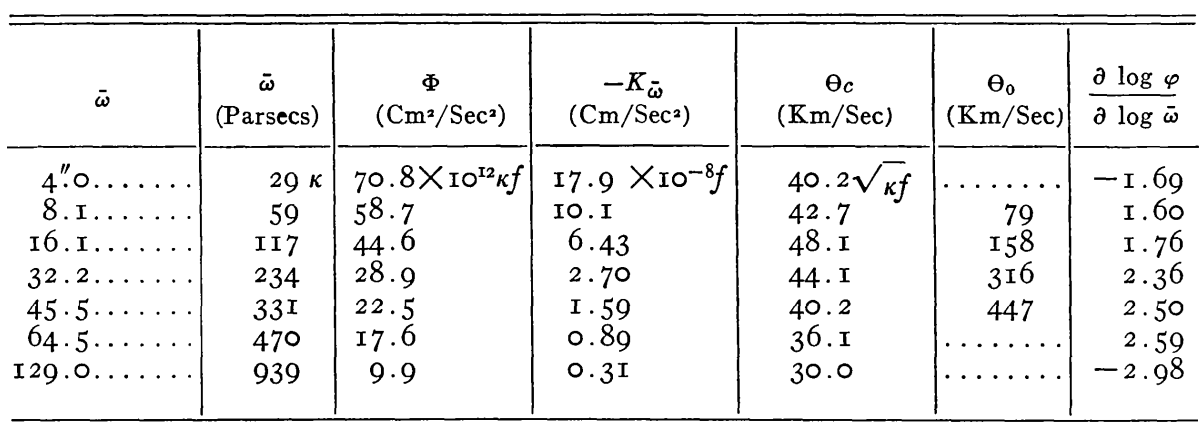

insight into the question of how far the most flattened elliptical nebulae and the regular cores of early-type spirals approach unstable configurations which could give rise to the formation of spiral arms. Humason's results on the rotation of $\mathrm{NGC}_{3}$ I I 5 seem to indicate, however, that there is not much prospect in this direction; not, at least, for this nebula.

Table 2 shows the values of the potential $\Phi$ and the acceleration $K_{\bar{\omega}}$ for points in the equatorial plane of the nebula, computed on the assumption that the distribution of density is the same as that of the light. The factor by which the true distance is larger than the distance of $\mathrm{I} .5$ million parsecs provisionally assumed is $\kappa ; f$ is the unknown ratio of mass density to light-density, the former being expressed in the mass of the sun per cubic parsec, the latter in the photographic light of the sun per cubic parsec as a unit (in the direct vicinity of the sun $f$ is about 2 ). The potential was assumed to be zero at infinity. 
The values of $\Phi$ and $K_{\bar{\omega}}$ were obtained by numerical integration with the aid of the equidensity surfaces given in Figure 7 , and with rough estimates about the more outlying surfaces. The surface corresponding to $\log \varphi=-2$.I $7\left(a=233^{\prime \prime}\right)$ was arbitrarily taken as the boundary of the nebula, the density being supposed to become zero outside this surface. The neglect of the material outside this surface is not likely to have had an appreciable influence on $K_{\bar{\omega}}$, but the values given for the potential will be too low. Accepting the approximate values given, the velocity of escape for $\bar{\omega}=4$ ". $\circ$ is found to be I I $\sqrt{\kappa f} \mathrm{~km} / \mathrm{sec}$; with $\kappa=\mathrm{I}$ and $f$ of the order of Ioo, it may roughly be estimated to be of the order of $\mathrm{I} 200 \mathrm{~km} / \mathrm{sec}$.

It is evident from the run of the values of $\Phi$ in Table 2 that, if the mass distribution in a system like NGC 3 II $_{5}$ is assumed to be like that of the light, such a system would be very stable. The condition for the appearance of dynamical instability is that at some place the force varies more rapidly than $\bar{\omega}^{-3}$, or the potential more rapidly than $\bar{\omega}^{-2}$. With the numbers given in Table 2 , the steepest variation of $\log \Phi$ with respect to $\log \bar{\omega}$ occurs in the outer parts of the nebula, but even at $\bar{\omega}=\mathrm{I} 20^{\prime \prime}$ we have only $\Phi \sim \bar{\omega}^{-0.86}$, that is, an even slower variation than in the case of a Newtonian field, so that the system would be very far from a state in which it could begin to develop spiral structure.

However, the spectrographic measures of rotation indicate that the distribution of mass is probably very different from that of the light; in such a case the stability cannot, of course, be judged from the distribution of the light. Humason's rotational velocities are given under $\theta_{0}$ in the sixth column of Table $2 .^{29}$ The measures represent the average radial velocity of the various parts of the nebula seen projected on top of each other; it is easily seen, however, that in a case like the present, where the rotational velocity increases linearly with the distance to the center, the observed radial velocity will be equal to the true rotational velocity at the corresponding point. Humason's measures extend to $45^{\prime \prime}$ from the center; on account of the small scale the measured velocity for $\bar{\omega}=8$ ". I is likely to have a considerable percentage of uncertainty.

It is interesting to compare the run of the rotational velocities with

${ }^{29}$ Report of the Director of Mt.W.Obs., 1936-37, p. 31. 
the entirely different run of the "circular" velocities (velocities corresponding to circular orbits) computed on the assumption that the mass distribution is the same as that of the light; these are given in the preceding column under $\Theta_{c}$ and appear to remain practically constant over the range in $\bar{\omega}$ considered. As $\theta_{0}$ will, in general, be lower than $\Theta_{c}$, we infer from the data for $\bar{\omega}=45^{\prime \prime} \cdot 5$ that, if $\kappa=\mathrm{I}$ (that is, if the distance is $\mathrm{I} \frac{1}{2}$ million parsecs), $f$ must be at least of the

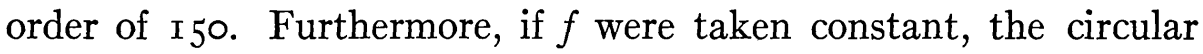
velocity at 8 ". I and I 6 ". I would come out many times larger than the rotational velocity. On the other hand, if we should assume that the rotational velocities are of the same order as the circular velocities, we should find that $f$ varies with $\bar{\omega}$ and that the distribution of the density differs radically from that of the light.

I believe it can be shown that the first alternative is very improbable. Let us assume for a moment that it were true; suppose, therefore, that $f$ be constant and, for instance, equal to $200 .^{30}$

The general relation between $\theta_{o}$ and $\theta_{c}$ in systems which are in a dynamically steady state may be written ${ }^{3 \mathrm{I}}$

$$
\theta_{c}^{2}-\theta_{0}^{2}=\overline{\Pi^{2}}\left[-\frac{\partial \log \varphi}{\partial \log \bar{\omega}}-\frac{\partial \log \overline{\Pi^{2}}}{\partial \log \bar{\omega}}-\left\{\mathrm{I}-\frac{\overline{\left(\Theta-\theta_{0}\right)^{2}}}{\overline{\Pi^{2}}}\right\}\right] . \text { (I) }
$$

Here $\Pi$ and $\theta$ denote the velocities in the direction of $\bar{\omega}$ and in that of the rotation; bars denote average values, thus $\bar{\theta}=\theta_{0}$. In the case of NGC 3 II 5 , where the angular velocity of rotation is approximately constant, the term $\left\{\mathrm{I}-\overline{\left(\theta-\theta_{0}\right)^{2}} / \bar{\Pi}^{2}\right\}$ is probably small; in the accompanying tentative calculations it has been neglected.

The seemingly perfect regularity and symmetry of the system suggests an approximately steady state. Jeans ${ }^{32}$ has shown that in the case of strict dynamical equilibrium the velocity distribution in the $\Pi$-direction must be identical with that in the $Z$-direction (parallel to the axis of rotation), so that

$$
\overline{Z^{2}}=\overline{\Pi^{2}} \text {. }
$$

${ }^{30}$ The total mass of the system would then be $7 \times \mathrm{ro}^{\mathrm{r}} \mathrm{solar}$ masses.

${ }^{3 x}$ The equation can, for instance, be derived directly from equation (10) given by Jeans in $M . N ., 82, \mathrm{I} 22, \mathrm{I} 922$.

${ }^{32}$ See, for instance, $M . N ., 76,8$ I, Igr 5 . 
Although it is by no means clear how such an equality of the two distributions would be established ${ }^{33}$ - for during an interval of $10^{\text {I0 }}$ or IO ${ }^{\text {II }}$ years the transfer of energy between individual stars in NGC 3 II 5 must have been negligible - I shall nevertheless use the above equation as a tentative working hypothesis for the following calculations. It should be noted that, as far as an estimate of the line width can be made, it indicates, according to information from Humason, that the dispersion of the $\Pi$-velocities cannot be much larger than about $100 \mathrm{~km} / \mathrm{sec}$ - an amount, therefore, which is of the same order as that computed for the velocities in the $Z$-direction (see Table 5).

If the gravitational force is known, the distribution of velocities in the $Z$-direction can be derived from the density distribution. In the computations I have assumed that the distribution of velocities could be represented as the sum of a number of exponential distributions, as follows:

$$
f(Z)=\sum \theta_{i} \frac{l_{i}}{\sqrt{\pi}} e^{-l_{i}^{2} Z^{2}}
$$

in which $\sum \theta_{i}=$ I. We have then the following equation, ${ }^{34}$ in which $K_{z}$ represents the gravitational acceleration

$$
\frac{\varphi_{z}(\bar{\omega})}{\varphi_{0}(\bar{\omega})}=\sum \theta_{i} e^{2 l_{i}^{2} \int_{0}^{z} K_{z} d z}
$$

The mean square velocity for a point near the equatorial plane is given by

$$
\overline{Z^{2}}=\sum \frac{\theta_{i}}{2 l_{i}^{2}}
$$

The computation of this mean square velocity might also have been made with the aid of the more general formula

$$
\overline{Z^{2}}=-\frac{\mathrm{I}}{\varphi_{0}} \int_{0}^{\infty} \varphi_{z} K_{z} d z
$$

which, however, would have necessitated extrapolations to infinity.

33 I am indebted to Dr. Woltjer for a discussion on some of the difficulties involved in introducing this equation. Cf. B.A.N., No. 238, p. 253.

${ }^{34}$ Cf. ibid. 
In the hypothetical case considered above, where the distribution of mass is the same as that of light and the ratio of mass to light is 200, $K_{z}$ can be directly computed from the known light-distribution. Somewhat approximate ${ }^{35}$ calculations were made for two regions of the nebula, namely, at $\bar{\omega}=\mathrm{I} 6{ }^{\prime \prime}$ I and $322^{\prime \prime} .2$, respectively. The results are shown in Table 3 (units $\mathrm{cm} / \mathrm{sec}^{2}$ and $\mathrm{cm}^{2} / \mathrm{sec}^{2}$, respectively; $\kappa$ was assumed to be I).

From these data it was found that the velocity distribution for $\bar{\omega}=\mathrm{I} 6$ ". I could be well represented as the sum of two Gaussian distributions with mean square velocities of 160 and $335 \mathrm{~km} / \mathrm{sec}$ and

TABLE 3

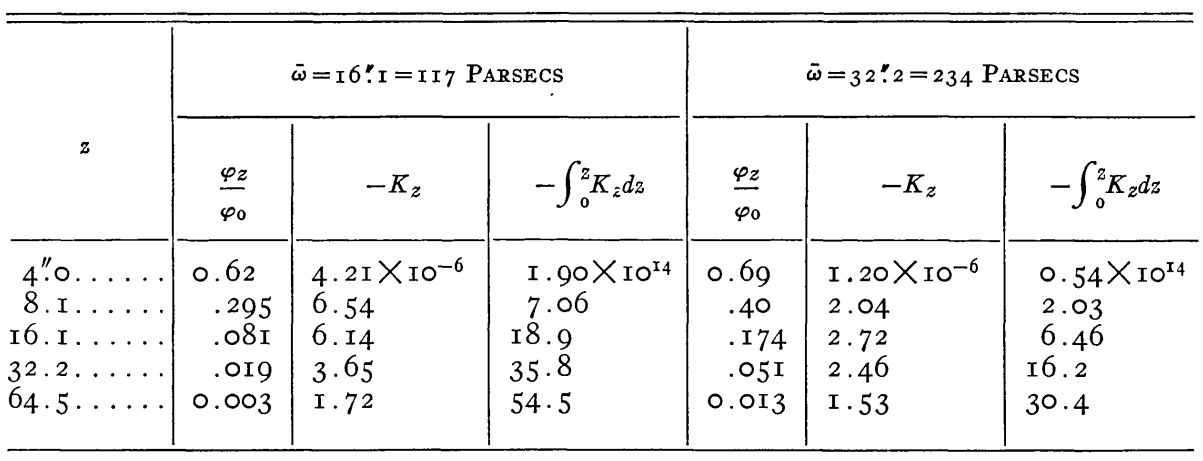

with $\theta_{\mathrm{r}}=0.52$ and $\theta_{2}=0.48$, so that the total mean square velocity $\sqrt{\overline{Z^{2}}}=259 \mathrm{~km} / \mathrm{sec}$. For $\bar{\omega}=32^{\prime \prime}$. 2 we obtain also a sum of two Gaussian distributions, corresponding to mean square velocities of 95 and $280 \mathrm{~km} / \mathrm{sec}$ with $\theta_{\mathrm{x}}=0.60$ and $\theta_{2}=0.40$; the total mean square velocity is $192 \mathrm{~km} / \mathrm{sec}$.

We can now insert these values of $\overline{Z^{2}}$ in formulae (2) and (I) and compute $\theta_{c}$ from the latter. As has been explained, the last term in the parentheses was neglected. From the values of $\overline{Z^{2}}$ just found, $\partial \log \bar{\Pi}^{2} / \partial \log \bar{\omega}$ was estimated to be about $-0.86 ; \partial \log \varphi / \partial \log \bar{\omega}$, which has been computed from the data on the light-distribution, is given in the last column of Table 2. In this way we find $\Theta_{c}=448$ and $468 \mathrm{~km} / \mathrm{sec}$ for $\bar{\omega}=\mathrm{I} 6$ ". I and 32 ". 2 , respectively. These values differ very considerably from the values 676 and $620 \mathrm{~km} / \mathrm{sec}$ computed

35 The approximation consisted in replacing the real equidensity surfaces by spheroids, which were so chosen that their volumes as well as axial ratios equaled those of the corresponding equidensity surfaces. 
from the light-distribution. Such high values for the circular velocity could have been obtained only by using very much larger values (between 400 and $500 \mathrm{~km} / \mathrm{sec}$ ) for the mean square peculiar velocities, values which would have been in contradiction with the distribution of light in the z-direction. Probably such high values as 400 or $500 \mathrm{~km} / \mathrm{sec}$ for the mean peculiar velocities could likewise be dismissed on the ground that the widths of the spectral lines would then have had to be greater than those observed. It may be concluded that the distribution of mass in the system must be considerably different from that of the light. A study of the light-distribution does not, therefore, enable us to draw conclusions regarding such problems as stability or the total mass of the system.

The question now arises: How must the distribution of mass density be in order that both the observed distribution of light and the measured rotational velocities as well as the conditions for dynamical equilibrium can be satisfied? The available data are not sufficient to permit a complete answer. We can start from attracting masses of various degrees of flattening. Detailed calculations have been made only for a model in which the equidensity surfaces in the attracting body are ellipsoids of axial ratio $c / a=2 / 3$. The high value $2 / 3$ was selected in order to be safely above the limit $0.55^{2}$, where the instability of a homogeneous mass sets in. The computations were made for three points, at $\bar{\omega}=8$ ". I, I 6 ". I, and $455^{\prime \prime} .5$, respectively; the gravitating mass was supposed to consist of an ellipsoid shell I with semimajor axis $a=\mathrm{Io}^{\prime \prime}$, an ellipsoidal shell II extending from $a=$ Io ${ }^{\prime \prime}$ to $a=20^{\prime \prime}$, and another shell III from $a=20^{\prime \prime}$ to $a=7 \mathrm{O}^{\prime \prime}$, outside of which the density was assumed to be zero. The densities in the inner ellipsoid and in the two shells were now determined in such a way that for the three points mentioned the equations (r), (2), and (4) were satisfied. The results are in Tables 4 and 5 .

The mass densities and light-densities are expressed in terms of the sun per cubic parsec. It will be noted that throughout the nebula the mass density is extremely high. Beyond $a=\mathrm{IO}^{\prime \prime}$ a value of I4O solar masses per cubic parsec is found, roughly equivalent to $10^{-20}$ $\mathrm{gm} / \mathrm{cm}^{3}$, or nearly 2000 times the density near the sun. The total mass of the system would become $5 \times 10^{10}$ solar masses. As the extent of the outer ellipsoid is entirely arbitrary, the real mass may differ 
considerably from this figure; the system may easily be as massive as the galactic system, the mass of which is estimated to be between IO ${ }^{\text {II }}$ and $2 \times 10^{\text {II }}$.

The most remarkable feature is that the mass density appears to remain practically constant outside $a=10^{\prime \prime}$, though the light-in-

TABLE $4^{*}$

Distribution OF MASS AND LIGHT IN NGC 3 II 5

\begin{tabular}{|c|c|c|c|c|}
\hline Shell & $a$ & $\begin{array}{c}\text { Mass } \\
\text { Density }\end{array}$ & $\begin{array}{c}\text { Light-Density } \\
\text { at } z=0\end{array}$ & $\frac{\text { Mass }}{\text { Light }}=f$ \\
\hline $\begin{array}{l}\text { I. } \ldots \ldots \cdots \cdots \\
\text { II } \ldots \ldots \cdots \cdots \\
\text { III } \ldots \ldots \ldots \ldots\end{array}$ & $\begin{array}{l}\mathrm{O}^{\prime \prime}-\mathrm{10} \mathrm{O}^{\prime \prime} \\
10-20 \\
20-70\end{array}$ & $\begin{array}{l}365 / \kappa^{2} \\
\mathrm{I} 44 / \kappa^{2} \\
\mathrm{I} 38 / \kappa^{2}\end{array}$ & $\begin{array}{ll}a=7^{\prime \prime} .5 & \mathrm{I} 8.6 / \mathrm{k}^{3} \\
a=\mathrm{I} 5.0 & 6.02 / \mathrm{k}^{3} \\
a=45.0 & 0.56 / \mathrm{k}^{3}\end{array}$ & $\begin{array}{r}19.6 \kappa \\
23.9 \kappa \\
249.0 \kappa\end{array}$ \\
\hline
\end{tabular}

* The symbol $\kappa$ is the factor by which the true distance differs from the assumed distance of $1.5 \times 10^{6}$ parsecs.

TABLE 5

\begin{tabular}{|c|c|c|c|c|c|c|}
\hline & \multicolumn{2}{|c|}{$\bar{\omega}=8: \mathrm{I}=59$ PaRSECS } & \multicolumn{2}{|c|}{$\bar{\omega}=I 6 ! I=I I 7$ PARSECS } & \multicolumn{2}{|c|}{$\bar{\omega}=45{ }^{\prime} 5=33$ I PARSECS } \\
\hline & $\begin{array}{c}\mathrm{I} / l \sqrt{2} \\
\mathrm{Km} / \mathrm{Sec}\end{array}$ & $\theta$ & $\begin{array}{c}\mathrm{I} / l \sqrt{2} \\
\mathrm{Km} / \mathrm{Sec}\end{array}$ & $\theta$ & $\begin{array}{c}\mathrm{I} / \mathrm{l} \sqrt{2} \\
\mathrm{Km} / \mathrm{Sec}\end{array}$ & $\theta$ \\
\hline $\begin{array}{l}\sqrt{\overline{\bar{Z}^{2}}} \ldots \ldots \ldots \\
\theta_{0} \ldots \ldots \ldots \\
\theta_{c} \ldots \ldots \ldots\end{array}$ & $\begin{array}{r}52 \\
104 \\
209 \\
418 \\
94 \\
79 \\
137\end{array}$ & $\begin{array}{r}0.46 \\
.50 \\
.035 \\
0.005 \\
\ldots \ldots \ldots \\
\ldots \ldots \ldots \\
\ldots \ldots \ldots\end{array}$ & $\begin{array}{r}45 \\
91 \\
\text { I } 82 \\
364 \\
\text { IO6 } \\
\text { I } 58 \\
\text { I } 97\end{array}$ & $\begin{array}{c}0.49 \\
.38 \\
.087 \\
0.034 \\
\ldots \ldots \ldots \\
\ldots \ldots \ldots \\
\ldots \ldots\end{array}$ & $\begin{array}{r}43 \\
85 \\
\text { I } 70 \\
340 \\
\text { I } 75 \\
447 \\
48 \mathrm{I}\end{array}$ & $\begin{array}{r}0.30 \\
.25 \\
.27 \\
0.18 \\
\ldots \ldots \ldots \ldots \\
\ldots \ldots \ldots \ldots\end{array}$ \\
\hline$\frac{\partial \log \overline{Z^{2}}}{\partial \log \bar{\omega}}$ & \multicolumn{2}{|c|}{ to.19 } & \multicolumn{2}{|c|}{$+0.5^{2}$} & \multicolumn{2}{|c|}{$+\mathrm{r} .46$} \\
\hline
\end{tabular}

tensity falls off steeply. The strongly condensed luminous system appears imbedded in a large and more or less homogeneous mass of great density. This is a feature which, to some extent, might be expected at once from a superficial consideration of Humason's result that the nebula rotates like a solid body; for, if the peculiar velocities are moderate, so that, except near the center, the measured rotational velocity resembles the circular velocity, Humason's re- 
sult means that the attracting mass must approximately be a homogeneous ellipsoid enveloping the measured region.

It may be noted that the mean square velocities, as shown in the fourth line from the bottom of Table 5 , are still high, though they are considerably smaller than in the model considered previously. A notable feature is the pronounced non-Gaussian character of the distribution of the velocities $Z$, which suggests that we are looking at a mixture of very different types of stars, or of stars and interstellar material. Though the particular velocity distributions found depend to a considerable extent on the assumptions concerning the mass distribution, we have seen that the non-Gaussian character remains, even if the mass distribution is supposed to be the same as the lightdistribution.

A very rough calculation along the same lines as that described but assuming axial ratios of $\frac{1}{3}$ instead of $\frac{2}{3}$ for the attracting ellipsoids indicates that in this case also the densities in the outer ellipsoids would come out nearly equal; the absolute densities as well as the values of $f$, however, would become more than twice as high as those found previously, while the peculiar velocities would also increase by a factor of nearly 2 . The mean square velocity at $\bar{\omega}=$ $45^{\prime \prime} .5$ would become of the order of $300 \mathrm{~km} / \mathrm{sec}$, a value so large that it could probably be verified from observations.

In the outer parts of the nebula the ratio $f$ of mass density to lightdensity is found to be very high; and this conclusion holds for whatever dynamical model we consider. The spectrum of the nebula shows the characteristics of G-type dwarfs. Since $f$ cannot be much larger than I for such stars, they can account for roughly only $\frac{1}{2}$ per cent of the mass; the remainder must mainly consist either of extremely faint dwarfs having an average ratio of mass to light of about 200 to I or else of interstellar gas or dust.

Let us first consider these latter two possibilities. If the mass consisted mainly of gas, the optical thickness for rays coming from the nucleus would be of the order of roo times the average optical thickness traversed by the light of a star at rooo parsecs distance in the galactic plane. The presence of layers of gas of such thickness might be verified from suitable spectra.

Many objects of the apparently more or less related type of Sa 
spirals show strong evidence of absorption effects. Though no direct trace of such effects can be found in NGC 3 I I 5 , the possibility cannot be excluded that there would be considerable amounts of solid interstellar material distributed evenly through the nebula. At the same time, however, it must be observed that if this matter is at all like the absorbing matter in the galactic system, it can at most account for a negligible fraction of the total density in NGC 3 II 5 , which is at least 3000 times that of the interstellar material in the galaxy. For, if the great density in the nebula were due to such absorbing matter, the transparency would be so low that we could not penetrate more than a very few parsecs into the system. ${ }^{36}$

The high value of $f$ found stands in sharp contrast to what is found in the galactic system. In the vicinity of the $\operatorname{sun} f$ is about 2; it may rise to, at most, 7 at large distances from the galactic plane. ${ }^{37}$ The ratio for the bulk of the galactic system can hardly be very much larger. A minimum value for the total amount of light contained in the part within the sun's distance from the center may be estimated by assuming that the light-density within an ellipsoid having a semimajor axis equal to the distance of the sun from the center (adopted as 8000 parsecs) and an axial ratio $c / a=\mathrm{I} / 20$, is everywhere the same as that observed near the sun, i.e., 0.048 times the photographic light of the sun per cubic parsec. The total amount of light thus found is $0.5 \mathrm{I} \times 1 \mathrm{I}^{\mathrm{ro}}$ units. From the rotation of the galaxy the total mass within the sun's distance from the center may be estimated as $9 . \mathrm{I} \times \mathrm{IO}^{10}$, so that we should find $f=\mathrm{I} 8$. The true value of $f$ must be expected to be considerably smaller, as the light will probably be concentrated toward the center, so that the above estimate would give too small a value for the total light. We may conclude that the ratio between mass and light for the galactic sys-

${ }^{36} \mathrm{It}$ is conceivable that an absorption might exist which is sufficiently powerful to reduce the light of the stars in the system by a considerable factor and that, thus, the real ratios $f$ for the undimmed light would be much smaller than those inferred from the present discussion. Such absorption would, however, also affect the central regions and, if called in to explain the high $f$-values in the outer region, would give impossibly low values for the central parts.

${ }^{37}$ Cf. B.A.N., No. 238 , p. 285 , 1932. It should be noted that the unit of light in that article was a star of +6.0 photographic absolute magnitude; the light densities given should therefore be multiplied by a factor of 0.506 to be reduced to the present units. 
tem as a whole is probably not much larger than that found in our surroundings, and is certainly very much smaller than that in NGC 3 II 5 .

The smallness of the dispersion in the radial velocities of nebulae in the Large Magellanic Cloud ${ }^{38}$ indicates that the mass of this system can hardly exceed $2 \times 10^{9}$; since the luminosity is about $3 \times 10^{8}$ times that of the sun, $f$ would be 6 at most - again very much smaller than the values found in the outer regions of NGC 3 I I 5 :

No reliable data for other systems have yet been published. It should be noted, however, that measures by Horace Babcock ${ }^{39}$ of the rotational velocity in the outer regions of the great Andromeda nebula indicate that the average value of $f$ for this system would be about 55 .

The dynamical models discussed do not give a direct explanation of the sharp edges of the isophotal surfaces of NGC 3 II 5 near its equatorial plane. The most probable explanation would seem to be that these are due to the admixture of a relatively small fraction of stars with low velocities.

In concluding this reconnaissance of the possible dynamical pe-

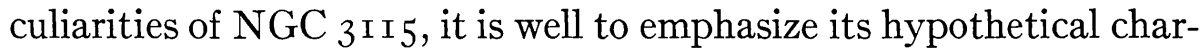
acter. Of the hypotheses underlying the analysis the most uncertain is no doubt the neglect of absorption within the nebula and the supposition that the light we observe comes directly from stars. If important absorption and dispersion of light has occurred, this would probably have tended to lessen the light-gradients; the true disparity between the distribution of luminous stars and of mass would then be still greater than that indicated. The numerical results for the velocity distributions would, however, lose their significance. The possibility that the equatorial plane of the nebula may be somewhat inclined to the line of sight also introduces some uncertainty; however, the sharpness of the edges of the isophotes near the equator shows that the inclination can hardly exceed $5^{\circ}$. The principal excuse for attempting an analysis in the present stage is that it may give some indication of what kind of additional observations would seem most promising for a better understanding of the struc-

${ }^{38}$ R. E. Wilson, Lick Obs. Pub., ז3, 189, I91 7.

${ }^{39}$ To be published in the Lick Obs. Bull. 
ture of a system of this kind. There cannot be any doubt that an extension of the measures of rotation to greater distances from the nucleus would be of exceptional interest. Also, our knowledge of the system could certainly be greatly advanced if it were possible to obtain spectroscopic estimates of the true mean peculiar velocities.

I am greatly indebted to Dr. Adams, who permitted the use of the 6o-inch telescope for the work on the light-distribution in nebulae. Likewise, my sincere thanks are due to Dr. Oosterhoff for his willingness to take the plates. To Mr. Humason I am indebted for showing me unpublished details of his measures of the rotation of $\mathrm{NGC}_{3}$ I I $5 ._{5}$ Professor Ornstein kindly permitted us to use the Moll registering photometer of the physical laboratory at Utrecht. Throughout, Messrs. Kleibrink and Pels have most efficiently co-operated in the measurements and computations.

Astronomical ObServatory

LeIden, Holland

October 23, 1939 